www.nature.com/pcan

\title{
Editorial
}

\section{Is the PSA test useless?}

\author{
Prostate Cancer and Prostatic Diseases (2004) 7, 271-272. doi:10.1038/sj.pcan.4500777
}

The clinical utility of serum prostate-specific antigen (PSA) testing for the early detection of prostate cancer has recently become one of the most contentious issues in urology. ${ }^{1,2}$ In the October 2004 issue of the Journal of Urology, Dr Thomas Stamey reports that the close relationship between serum PSA values and tumour volume at the time of radical prostatectomy appears to have been lost, probably as a result of PSA screening. ${ }^{3}$ This has led to headlines in the media along the lines that the 'PSA test is useless'. To add to the concerns of urologists and their patients, a recent publication by Thompson et $\mathrm{l}^{4}$ reveals that a substantial number of men harbour adenocarcinoma in spite of having a PSA value $<4.0 \mathrm{ng} / \mathrm{ml}$. Using the placebo arm of a randomised study of the use of finasteride to prevent the development of prostate cancer ${ }^{5}$ as the study group, these investigators found that $15.2 \%$ of these 2950 men were found to harbour prostate tumours, $14.9 \%$ of which had a Gleason score of 7 or higher. The relationship between the PSA value and the risk of adenocarcinoma still seems to hold, and those with higher PSA values appear to have a greater risk of higher grade and stage cancers. However, there is considerable overlap in the distributions among grades. A small but significant number of patients had higher grade cancers even though their PSA value was extremely low.

What are the implications of these somewhat unexpected findings? The current practice of reassuring men whose PSA is below $4.0 \mathrm{ng} / \mathrm{ml}$ that they are unlikely to harbour prostate cancer no longer appears appropriate. The initial reaction to these new data might be to advocate an across the board reduction in the PSA cut point, which indicates prostate biopsy to $2.5 \mathrm{ng} / \mathrm{ml}$. However, this manoeuvre would undoubtedly increase the detection of prostate cancer, it would also heighten the risk of detection of clinically insignificant tumours. Furthermore, even a cut-point of $2.5 \mathrm{ng} / \mathrm{ml}$ would risk missing detection in a number of patients who turn out to have prostate cancer in spite of having a PSA of $<2.5 \mathrm{ng} / \mathrm{ml}$.

The central problem for those who advocate the early detection of prostate cancer is the lack of firm evidence that such a manoeuvre actually reduces the risk of death from the disease. Evidence on this crucial issue will not be forthcoming for some time yet. Data from both the European Randomised Study of Prostate Cancer Screening and PLC study from the USA are not expected until at least 2008. In the meantime, we have to depend on indirect and inconclusive evidence.

Two important data sets have been published recently, which are relevant in this context. Firstly, Holmberg et al have confirmed that radical prostatectomy reduces the risk of development of metastases by around $50 \%$. Secondly, Johansson et $a l^{6}$ have reported that patients with prostate cancer who are managed by watchful waiting have a substantial mortality even as long as
$15-20 y$ after the initial diagnosis. Follow-up at this stage revealed a substantial reduction in progression-free survival (from 45-36\%), survival without metastases (from 76.9 to $51.2 \%$ ) and prostate cancer-specific survival (from 78.7 to $54.4 \%$ ). The prostate cancer mortality rate increases from 15 per 1000 person-years during the first 15 years to 44 per 1000 person years beyond 15 years of follow-up. Clearly, many of these deaths, and the morbidity that preceded them, could most likely have been averted by judicious and effective earlier intervention. These new data provide the rationale for more aggressive management strategies in prostate cancer patients with longer life expectancies.

So what should we now say to men concerned that they may be suffering from localised prostate cancer and who wish to avoid developing advanced disease in the future? First and foremost, the uncertainties about prostate cancer diagnosis and treatment need to be clearly and concisely explained. Second, PSA, while remaining a useful, but imperfect, predictor of prostate cancer (the higher the PSA, the greater the risk that prostate cancer may be present), cannot completely exclude clinically significant prostate cancer. Currently, transrectal ultrasound-guided biopsy of the prostate is the only reliable way to accomplish this. Third, even if cancer is diagnosed, not every patient requires treatment. Active surveillance should certainly be discussed as an option in patients with lower Gleason scores and smaller volume tumours, before more aggressive treatment options are pursued.

Fortunately, just as the shine appears to be fading on PSA as a tumour marker, at least in terms of its ability to rule out prostate cancer conclusively, other alternatives are appearing on the horizon. For example, DD3 ${ }^{\text {PCA3 }}$ is the most prostate cancer-specific gene described to date. $^{7}$ A time-resolved fluorescence-based, quantitative RT-PCR analysis has been developed. Prostate tumours showed a 66-fold upregulation of $\mathrm{DD}^{\mathrm{PCA} 3}$ when compared with benign prostatic tissue. ${ }^{8}$ This upregulation was found in more than $95 \%$ of prostate cancer specimens studied. Therefore, this DD3 ${ }^{\text {PCA3}}$-based RTPCR assay was used for the identification of prostate cancer in urine sediments obtained after prostatic massage. In a cohort of 108 men with a PSA value of $>3.0 \mathrm{ng} / \mathrm{ml} 24$ were shown to have prostate cancer on biopsy. Of these 24 men, 16 were shown to be positive for DD3 ${ }^{\text {PCA3 }}$, indicating a sensitivity of $67 \%$. Furthermore, a negative value of $90 \%$ was calculated. Clearly, a multicentre study using this RT-PCR assay will be required to verify these data and evaluate the clinical utility of this molecular diagnostic test. However, this new test appears at this stage to have the potential to improve the specificity of diagnosis and thus reduce significantly the number of unnecessary prostate biopsies required to diagnose this most common cancer of men. ${ }^{9}$ 
The above dilemma exemplifies rather well how current research is needed to move many of these very difficult arguments forward. In this fourth issue of Volume 7 of Prostate Cancer and Prostatic Diseases, many of the problems that we as clinicians face on a day to day basis are addressed and prospects for the future are set out. We again thank our contributors for their efforts and you the reader for your support. Much remains to be done on behalf of our patients with prostate disease to resolve the problems that surround screening, diagnosis and treatment. It is the intention of the editors of this journal to remain at the forefront of these ongoing efforts.

R Kirby and JW Moul

Editors

\section{References}

1 Boyle P. Screening for prostate cancer: have you had your cholesterol measured? BJU Int 2003; 92: 191-199.
2 Neal DE, Donovan JL. Prostate cancer: to screen or not to screen? Lancet Oncol 2000; 1: 17-24.

3 Stamey TA et al. The prostate specific antigen era in the United States is over for prostate cancer: what has happened in the last 20 years? J Urol 2004; 172: 1297-1301.

4 Thompson IM et al. Prevalence of prostate cancer among men with a prostate-specific antigen level $<4.0 \mathrm{ng}$ per millilitre. N Engl J Med 2004; 350: 2239-2246.

5 Holmberg. A randomised trial comparing radical prostatectomy with watchful waiting in early prostate cancer. $N$ Engl J Med 2002; 347: 781-789.

6 Johansson J-E et al. Natural history of early localised prostate cancer. JAMA 2004; 291: 2713-2719.

7 Schalken JA, Hessels D, Verhaegh G. New targets for therapy in prostate cancer: differential display code 3 (DD3 $\left.{ }^{\mathrm{PCA} 3}\right)$, a highly prostate cancer-specific gene. Urology 2003; 62 (suppl 5A): $34-43$.

8 Hessels D et al. DD3 ${ }^{\mathrm{PCA} 3}$-based molecular urine analysis for the diagnosis of prostate cancer. Eur Urol 2003; 44: 8-16.

9 Tinzl M, Marberger M, Horvath S, Chypre C. DD3 ${ }^{\text {PCA3 }}$ RNA analysis in urine-a new perspective for detecting prostate cancer. Eur Urol 2004; 46: 182-187. 